\title{
Editorial
}

\section{Atrial Fibrillation, Past and Future: From a Stroke Non-Entity to an Over-Targeted Cause}

\author{
Louis R. Caplan \\ Department of Neurology, Beth Israel Deaconess Medical Center, Harvard Medical School, Boston, MA, USA
}

Internists, neurologists, strokeologists, and cardiologists are now heavily focused on identifying occult atrial fibrillation as a cause of brain ischemic events that have no clear cause even after initial investigations. Patients with brain ischemia (transient ischemic attacks [TIAs] and strokes) are almost universally subjected to cardiac rhythm monitoring while hospitalized, Holter monitoring as outpatients for days, and even implantation of monitors that detect cardiac rhythm abnormalities during a period of years. Device manufacturers are trying to make better noninvasive monitoring devices some connected to smart phones to detect atrial fibrillation. The intent is to record an episode, or episodes, of atrial fibrillation before the patient has a stroke. Those in whom atrial fibrillation is detected are considered for anticoagulation. Reports detail the results of this monitoring but many uncertainties remain. How long to monitor? What duration of an episode of atrial fibrillation is significant $10 \mathrm{~s}$, a min, or $6 \mathrm{~min}$ ? Is one episode enough or should there be at least several of them?

In this study, after briefly reviewing the history of atrial fibrillation and stroke, I discuss the issue of intensively targeting atrial fibrillation. Is it really the elephant in the

\section{KARGER}

(c) 2018 S. Karger AG, Basel

E-Mail karger@karger.com

www.karger.com/ced room - the only major culprit responsible for strokes of unknown cause? Instead, should we focus more on the abnormal function of the cardiac atria?

\section{Historical Background of the Development of Observations and Ideas}

Even in Osler's time (late 19th and early 20th centuries), cardiac diseases, especially rheumatic heart disease with mitral stenosis, and bacterial endocarditis have been well known to serve as sources of brain embolism. Around the midpoint of the 20th century, cardiologists began to posit that atrial fibrillation was an important predecessor of brain embolism in patients with rheumatic mitral stenosis [1]. In 1949, Miller Fisher was one of the first individuals to suggest a role for atrial fibrillation in causing brain embolism, even in the absence of rheumatic heart disease or endocarditis.

Fisher wrote: "I had the opportunity to examine the cerebral arteries before slicing 3 brains that had large hemorrhagic infarcts. The basal vessels were empty of thrombus. People were signing out these cases as cerebral artery thrombosis - but pathologically there 
was no thrombus. Afterwards, I looked up the records on these 3 cases and they had all been in atrial fibrillation and the general autopsy had shown infarcts in the spleen and kidneys" $[2,3]$.

During the early 1960s, Fisher told me that while having lunch with 3 cardiologists, he shared with them his observation that many subsequent necropsies had shown definite brain embolism in patients with atrial fibrillation but who had no valve disease. These very experienced cardiologists ignored the suggestion and replied that they had many atrial fibrillation patients and were aware of no strokes. (All 3, including the famous proponent of exercise Dr. Paul Dudley White, eventually developed atrial fibrillation and large brain emboli.)

In 1977, a necropsy study reported further evidence that lone atrial fibrillation (no accompanying valve disease) was an important cause of embolism [4].

"Atrial fibrillation is well known to increase greatly the risk of systemic arterial embolism in patients with mitral valve disease... a study was made of embolic occurrences in 333 autopsy patients with atrial fibrillation associated with various kinds of heart disease. Considering only symptomatic emboli with pathologic or surgical confirmation, embolism occurred in $41 \%$ of patients with mitral valve disease, $35 \%$ of those with ischemic heart disease, $35 \%$ of those with coexisting mitral and ischemic heart disease, and 17\% of those with 'other' types of heart disease. Embolism was found only in $7 \%$ of a control group of 58 autopsy patients with ischemic heart disease without atrial fibrillation. These findings suggest a high risk of embolism from atrial fibrillation of any origin" [4].

The very next year, epidemiological results from the Framingham Heart study confirmed a high frequency of embolism from non-valvular atrial fibrillation [5]. After 24 years of follow-up in the Framingham Heart Study, 345 strokes had occurred, 27 in those individuals with chronic atrial fibrillation -7 with Rheumatic Heart Disease and 20 with non-rheumatic atrial fibrillation. In those with atrial fibrillation and rheumatic heart disease, the incidence of stroke was increased 17.6 fold and in those with atrial fibrillation in the absence of valve disease, there was a 5.6-fold increased stroke incidence [5].

Miller Fisher, in 1979, published a plea for considering anticoagulation in patients with persistent or paroxysmal atrial fibrillation before brain ischemia occurred [6]. Fisher reviewed the hospital records of 100 patients who had atrial fibrillation (83 lone, 17 with valve disease). Totally, 71\% of the 109 episodes among the 100 patients resulted in death or in an unacceptable neurological deficit, emphasizing the severity of the initial stroke. He also reviewed random records of 100 patients who were hospitalized with atrial fibrillation but not anticoagulated; 35 had embolic events ( $86 \%$ brain, $14 \%$ systemic). Among 48 patients who did not have strokes initially, 35\% had typical embolic strokes during the 4 years of follow-up [6].

After echocardiography became available, follow-up of patients with non-valvular atrial fibrillation further documented a high frequency (10\%) of systemic embolism [7]. Echocardiograms sometimes showed thrombi in the left atrial appendage, large left atria, and occasional thrombi within the left atrium. By 1986, the Cerebral Embolism Task Force concluded, "Nonrheumatic nonvalvular atrial fibrillation is the most common cardiac condition associated with presumed embolic stroke, accounting for almost half of cardiogenic embolic strokes in recent clinical series" [8]. By the end of the 20[th] century, it was generally accepted that non-valvular atrial fibrillation was an important (perhaps the most important) cause of systemic and brain embolism. During the period between 1980 and 2000 , trials within the United States and international therapeutic trials studied the relative effectiveness and safety of placebo, aspirin, and warfarin in patients with known atrial fibrillation, some of whom had already had instances of brain ischemia. All documented the superior effectiveness of warfarin, a vitamin $\mathrm{K}$ antagonist, in preventing brain embolism in patients with non-valvular atrial fibrillation. More recently therapeutic trials have clearly shown that newer anticoagulants (NOACS; a direct thrombin inhibitor and 3 activated Factor $\mathrm{Xa}$ antagonists) are at least as effective as warfarin and cause less important brain bleeding.

\section{Present Generally Accepted Concepts Regarding Non-Valvular Atrial Fibrillation and Stroke}

1. Most atrial fibrillation-related brain infarcts are embolic, but some are a result of coexistent cardiovascular conditions (e.g., hypertension, cervicocranial atherosclerosis, etc.).

2. Atrial fibrillation is often first discovered when a stroke occurs.

3. The initial atrial fibrillation-related strokes are often large and devastating (cardiac source thrombi are larger than those that originate in the arteries supplying the brain and cardiac origin brain infarcts are larger than those caused by penetrating artery and large artery cervicocranial atherostenotic lesions).

4. Anticoagulation is effective in preventing embolism in atrial fibrillation patients but has important safety risks especially in the elderly, a group in whom atrial fibrillation is prevalent. Many atrial fibrillation patients are not anticoagulated mostly because of presumed risk of anticoagulation. 
5. In atrial fibrillation patients in whom benefit is judged to exceed risk, NOACS are preferred if affordable over vitamin $\mathrm{K}$ antagonists.

\section{Back to Basics-Why and How Does Atrial Fibrillation Relate to Brain Embolism?}

During atrial fibrillation, atrial and atrial appendage contractions are relatively inefficient; atrial emptying decreases and stasis of blood develops. Stasis and the coagulability of the blood interact to form red fibrin-erythrocyte rich thrombi that are ejected during ventricular contraction into the aorta and thence to systemic arteries including the brain. Dehydration and hypovolemia, platelet activation, genetic and acquired abnormalities of the coagulation cascade, and increases in acute phase reactants stimulated by infection, inflammation, and neoplasia, all enhance thrombus formation in the heart and systemic and intracranial veins.

If one accepts the logic and evidence for this conceptualization, then it follows that other contractile cardiac abnormalities also favor red thrombus formation in the heart. Atrial and ventricular dysfunction should also predispose to red thrombus formation. The sick-sinus syndrome has also been convincingly shown to be a risk for brain embolism. Left ventricular abnormalities, such as congestive heart failure with low ejection fractions, ventricular aneurysms, akinesia or hypokinesia of the ventricle or portions of the ventricle, can also predispose to red thrombus formation. Atrial morphological and functional abnormalities other than documented atrial fibrillation could, if severe enough, predispose to stasis of blood and in situ thrombus formation. Some atrial abnormalities are predictive of the development of atrial fibrillation. A recent study confirmed that runs of supraventricular tachycardia predispose to development of atrial fibrillation [9].

\section{Newer Diagnostic Methods and Previously Unrecognized Abnormalities}

Now the heart's morphology and functions can also be interrogated by using Cardiac MRI [10-12], Cardiac CT angiography [13], and Doppler monitoring of flow velocities [14, 15], and by histological analysis of atrial and ventricular biopsies [16], as well as by animal experimental studies $[17,18]$. Fibrosis in the atrium and ventricle can now be detected and quantified by Cardiac MRI [11], and by histological analysis $[16,17]$.

Atrial Fibrillation, Past and Future
Morphological and functional changes in the left atrium and left atrial appendage can presage atrial fibrillation but could also be sufficient to cause blood stasis and thrombus formation [19]. Ventricular abnormalities are also important to detect. The atrial abnormalities include: fibrosis and altered morphology such as important enlargement of the left atrium [20-22]; altered contractility with abnormal ejection velocities; mechanical discordance of the left atrium and its appendage [15]. In the Framingham Heart Study, left atrial enlargement, increased left ventricular wall thickness, and reduced left ventricular fractional shortening were predictive of the risk for nonrheumatic atrial fibrillation [22].

\section{Integrating Atrial and Ventricular Cardiopathy Data into Care of Patients with Brain Ischemia}

Classification of a brain infarct as embolic is based on the following factors: the size, location, and morphology of the infarct, the patient's risk factors, and the onset and evolution of the clinical symptoms and signs. Patients with brain ischemia (strokes [brain infarcts] and TIAs) can be practically characterized into 3 prototypes: penetrating artery disease; cardiogenic embolism; and large artery disease in the neck and/or intracranially often with intra-arterial embolism. The ASCOD phenotyping effectively characterizes these subtypes [23-25]. Describing pure typical prototypes is useful in simplifying their identification. ASCOD captures and weights the overlap between all of the underlying diseases present in ischemic stroke patients [25].

Treatment of patients with penetrating artery disease consists of risk factor (especially blood pressure) control and antiplatelet agents. It is uncertain if double antiplatelets are optimal, and which antiplatelets - aspirin and clopidogrel or aspirin and cilostazole - are effective and for how long. The role of statins is uncertain. Treatment of patients with cardiogenic embolism now involves risk factor control, statins, and anticoagulants (preferably NOACS). Those patients with large artery disease are treated with risk factor control, statins, and antiplatelets or surgery or stenting if the stenoses are severe and medical treatment fails.

Of course, many patients do not represent pure prototypes but have risk factors and cardiac and vascular imaging profiles that are non-diagnostic or are compatible with more than one etiology. In these patients, judgment and clinical experience are essential in choosing treatment. 


\section{Moving Forward}

Anticoagulants are generally regarded as the treatment of choice in patients with atrial fibrillation, whether or not they already have had brain ischemia, when there are no contraindications. I do not favor relying on the CHASDS or CHADS-VASC scores to decide on anticoagulation. These scores do predict stroke risks in patients with atrial fibrillation, but they also predict stroke risks equally in patients who do not have atrial fibrillation [26]. Many of the strokes in patients with atrial fibrillation are due to large and penetrating artery disease conditions in which anticoagulants are not recommended and this can incur additional risks.

Major areas of therapeutic uncertainty are:

1. Those patients whose brain imaging, risk factors, and clinical onset and course suggest an embolic stroke mechanism, but a presently acknowledged high-risk cardiac or arterial source is not apparent after the initial investigations. Hart et al. [27] have made a strong case for considering these patients a "new clinical construct". Randomized therapeutic trials are now ongoing concerning this group dubbed embolic strokes of uncertain source (ESUS). These patients now are almost always subjected to cardiac rhythm monitoring for variable periods to detect atrial fibrillation. Most patients who are not in these trials are now treated by their physicians with antiplatelet agents.

2. Patients with atrial and other non-valvular cardiopathies detected by modern cardiac evaluations who do not have recognized atrial fibrillation. These patients are now almost invariably monitored for the development of atrial fibrillation before prescribing anticoagulants. Most are treated with antiplatelet agents.

3. Young patients with PFOs with and without atrial septal aneurysms who do not have other cardiac or arterial sources of embolism.

4. Patients with atrial and other non-valvular cardiopathies other than PFOs who also have potential aortic or substenotic cervico-cranial arterial lesions that could serve as a source of brain embolism. These patients are now often monitored for the development of atrial fibrillation and are treated with antiplatelet agents.

I suggest that PFO group patients be considered separately. Many trials, past and ongoing, are testing optimal management. The other groups of ESUS patients should be entered into therapeutic trials whenever possible. Trials of anticoagulants vs. aspirin are ongoing and preliminary results have not shown a superiority of NOACS over aspirin. However, these trials lump all

ESUS patients who do not have atrial fibrillation, severe Carotid artery stenosis, or atrial thrombi together. These trials do not require or include analysis of cardiac abnormalities that can presage atrial fibrillation or themselves stimulate thrombus formation and embolization. It is important in my opinion to separate those patients with cardiac investigations that show atrial and other non-valvular cardiopathies into separate trials, since the likelihood of developing atrial fibrillation is high and the heart is the most likely source of embolism. Potential treatment groups in these newer trials are anticoagulants (NOACS), antiplatelets, and a combination of NOACs and antiplatelets. Aspirin and a NOAC have already been used in trials in patients with coronary artery disease and the combination of an anticoagulant with an antiplatelet seem relatively safe [28-30]. Clearly these trials are of high priority and importance.

In patients who are not entered into trials, I urge clinicians to avoid placing all their energy into monitoring for atrial fibrillation. They should not wait until atrial fibrillation is recognized to consider treatment with anticoagulants. Often the onset of atrial fibrillation is heralded by a large stroke. Clinicians should think carefully and thoroughly about the patients' risk factors, brain lesions, cardiac findings, and vascular imaging results. Are there cardiac lesions sufficient to result in stasis and red thrombus formation? In some patients who have brain ischemia (and perhaps even in those in whom brain ischemia is threatened), the presence of an atrial cardiopathy as detected by echocardiography, electrocardiography, and newer technologies, if severe enough, and when the benefit/risk profile for anticoagulation is satisfactory, should be treated with anticoagulants even though they have not been shown to have atrial fibrillation.

References

Cerebrovasc Dis 2018;45:149-153 DOI: $10.1159 / 000488063$
1 Harris AW, Levine SA: Cerebral embolism in mitral stenosis. Ann Intern Med 1941;15: 637-643.

2 Fisher CM: Memoirs of a Neurologist, vol 1 Rutland Vermont, Sharp \& Co., Printers, 2006.

13 Wolf PA: Awareness of the role of atrial fibrillation as a cause of ischemic stroke. Stroke 2014;45:e19-e21.

-4 Hinton RC, Kistler JP, Fallon JT, Friedlich $\mathrm{AL}$, Fisher CM: Influence of etiology of atrial fibrillation on incidence of systemic embolism. Am J Cardiol 1977;40:509513.

$\checkmark 5$ Wolf PA, Dawber TR, Thomas HE Jr, Kannel WB: Epidemiologic assessment of chronic atrial fibrillation and risk of stroke: the Framingham study. Neurology 1978;28: 973-977. 
-6 Fisher CM: Reducing risks of cerebral embolism. Geriatrics 1979;34:59-61, 65-66.

7 Cabin HS, Clubb KS, Hall C, Perlmutter RA, Feinstein AR: Risk for systemic embolization of atrial fibrillation without mitral stenosis. Am J Cardiol 1990;65:1112-1116.

8 Cardiogenic brain embolism. Cerebral Embolism Task Force. Arch Neurol 1986;43:7184.

-9 Weber-Krüger M, Lutz C, Zapf A, et al: Relevance of supraventricular runs detected after cerebral ischemia. Neurology 2017;89:15451552.

10 Daccarett M, McGann CJ, Akoum NW, MacLeod RS, Marrouche NF: MRI of the left atrium: predicting clinical outcomes in patients with atrial fibrillation. Expert Rev Cardiovasc Ther 2011;9:105-111.

11 Petersen SE, Aung N, Sanghvi MM, Zemrak F, Fung K, Paiva JM, et al: Reference ranges for cardiac structure and function using cardiovascular magnetic resonance (CMR) in Caucasians from the UK Biobank population cohort. J Cardiovasc Magn Reson 2017;19:18.

12 Yaghi S, Liberman AL, Atalay M, Song C, Furie KL, Kamel H, et al: Cardiac magnetic resonance imaging: a new tool to identify cardioaortic sources in ischaemic stroke. J Neurol Neurosurg Psychiatry 2017;88:31-37.

13 Hur J, Kim YJ, Lee HJ, et al: Left atrial appendage thrombi in stroke patients: detection with two-phase cardiac CT angiography versus transesophageal echocardiography. Radiology 2009;251:683-690.
14 Naqvi TZ, Zaky J: Electric dissociation within left atrial appendage diagnosed by Doppler echocardiography. J Am Soc Echocardiogr 2004;17:1077-1079.

15 Warraich HJ, Gandhavadi M, Manning WJ: Mechanical discordance of the left atrium and appendage: a novel mechanism of stroke in paroxysmal atrial fibrillation. Stroke 2014;45: 1481-1484.

16 Frustaci A, Chimenti C, Bellocci F, Morgante E, Russo MA, Maseri A: Histological substrate of atrial biopsies in patients with lone atrial fibrillation. Circulation 1997;96:1180-1184.

17 Boldt A, Wetzel U, Lauschke J, et al: Fibrosis in left atrial tissue of patients with atrial fibrillation with and without underlying mitral valve disease. Heart 2004;90:400-405.

18 Cai H, Li Z, Goette A, et al: Downregulation of endocardial nitric oxide synthase expression and nitric oxide production in atrial fibrillation: potential mechanisms for atrial thrombosis and stroke. Circulation 2002;106: 2854-2858.

19 Natale A, Jalife J: Atrial Fibrillation, from Bench to Bedside. Totowa NJ, Humana Press, 2008.

20 Kamel H, Okin PM, Elkind MSV, Iadecola C. Atrial Fibrillation and Mechanisms of Stroke. Stroke 2016;47:895-900.

21 Caplan LR, D’Cruz I, Hier DB, Reddy H, Shah S: Atrial size, atrial fibrillation, and stroke. Ann Neurol 1986;19:158-161.

22 Vaziri SM, Larson MG, Benjamin EJ, Levy D: Echocardiographic predictors of nonrheu- matic atrial fibrillation. The Framingham Heart Study. Circulation 1994;89:724-730.

23 Amarenco P, Bogousslavsky J, Caplan LR, Donnan GA, Hennerici MG: New approach to stroke subtyping: the A-S-C-O (phenotypic) classification of stroke. Cerebrovasc Dis 2009;27:502-508.

24 Amarenco P, Bogousslavsky J, Caplan LR, Donnan GA, Hennerici MG: Classification of stroke subtypes. Cerebrovasc Dis 2009;27: 493-501.

25 Amarenco P, Bogousslavsky J, Caplan LR, Donnan GA, Wolf ME, Hennerici MG: The ASCOD phenotyping of ischemic stroke (Updated ASCO Phenotyping). Cerebrovasc Dis 2013;36:1-5.

26 Ntaios G, Lip GY, Makaritsis K, et al: $\mathrm{CHADS}_{2}$, $\mathrm{CHA}_{2} \mathrm{~S}_{2} \mathrm{DS}_{2}$-VASc, and long-term stroke outcome in patients without atrial fibrillation. Neurology 2013;80:1009-1017.

27 Hart RG, Diener HC, Coutts SB, et al; Cryptogenic Stroke/ESUS International Working Group: Embolic strokes of undetermined source: the case for a new clinical construct. Lancet Neurol 2014;13:429-438.

28 Mega JL, Braunwald E, Wiviott SD, et al: Rivaroxaban in patients with a recent acute coronary syndrome. N Eng J Med 2012;366:9-19.

29 Eikelboom JW, Connolly SJ, Bosch J, et al: Rivaroxaban with or without aspirin in stable cardiovascular disease. N Engl J Med 2017; 377:1319-1330.

30 Braunwald E: An important step for Thrombocardiology. N Engl J med 2017;377:1387-1388. 